Grupos de interés e impuesto al consumo de bebidas azucaradas en Colombia

Juliana Díaz-García, Germán Valencia-Agudelo, Isabel Cristina Carmona-Garcés y Laura Inés González-Zapata 
Juliana Diaz-García, Germán Valencia-Agudelo, Isabel Cristina Carmona-Garcés y Laura Inés González-Zapata

Grupos de interés e impuesto al consumo de bebidas azucaradas en Colombia Resumen: Los grupos de interés son actores clave en las decisiones de política pública. Este articulo analiza el proceso decisorio de la propuesta de impuesto al consumo de bebidas azucaradas en Colombia y el papel de grupos de interés en ese proceso. Con este objetivo, el presente estudio usa el análisis stakeholder, para el cual se recolectó información sobre el proceso decisorio y el papel de los grupos de interés a partir de revisión documental y entrevistas estructuradas; además, se determinó el grado de relaciones, incidencia y poder de los grupos de interés entrevistados. Los hallasgos muestran la influencia y el poder de la industria de bebidas para bloquear procesos de agenda pública que van en contra de sus intereses; para lo cual usan diferentes instrumentos, como la capacidad organizativa y de persuasión, su poder económico, entre otros. En conclusión, la toma de decisiones se da en función de la movilización de los distintos intereses sectoriales. Este caso refleja la necesidad del fortalecimiento del sistema democrático, con mayor incidencia de la sociedad civil.

Palabras clave: grupos de interés; poder politico; impuestos; bebidas no alcobólicas.

Clasificación JEL: D78, H3, I18.

\section{Stakeholders and the consumption tax of sugar-sweetened beverages in Colombia}

Abstract: Stakeholders are key actors in public policy decisions. This article analyzes the decisionmaking process of the proposed consumption tax on sweetened beverages in Colombia and the role of stakeholders in that process. To this purpose, this study uses stakeholder analysis, for which information on the decision-making process and the role of stakeholders was collected from document review and structured interviews; in addition, the level of relationships, incidence, and power of the interviewed stakeholders was determined. The findings show the influence and power of the beverage industry to block public agenda processes that go against their interests; for which they use different instruments, such as organizational and persuasive capacity, their economic power, among others. In conclusion, decision making is a function of the mobilization of different sectoral interests. This case reflects the need to strengthen the democratic system, with greater influence from civil society.

Keywords: stakeholders; political power; taxes; non-alcoholic beverage.

\section{(cc) BY-NC-SA}

Este artículo y sus anexos se distribuyen por la revista Lecturas de Economía bajo los términos de la Licencia Creative Commons Atribución-NoComercial-CompartirIgual 4.0. https://creativecommons.org/licenses/by-nc-sa/4.0/ 


\section{Groupes d'intérêt et taxe à la consommation sur les boissons sucrées en Colombie}

Résumé: Les groupes d'intérêt sont des acteurs clés dans les décisions concernant les politiques publiques. Cet article analyse le processus décisionnel du projet de loi qui prévoit une taxe à la consommation sur les boissons sucrées en Colombie, ainsi que le rôle joué par les groupes d'intérêt. Cette étude utilise une analyse des parties prenantes, dont les informations sur le processus décisionnel et le rôle des groupes d'intérêt ont été collectées d'après une revue documentaire et d'entretiens structurés. De plus, le degré de relations, l'incidence et le pouvoir des groupes d'intérêt interrogés ont été ainsi déterminés. Les résultats montrent l'influence et le powvoir de l'industrie des boissons pour bloquer les processus d'agenda public qui vont à l'encontre de l'intérêt général. Cette industrie utilise différents instruments tels que la capacité organisationnelle, la persuasion et le pouvoir économique parmi d'autres. En conclusion, la prise de décision se fait sur la base de la mobilisation des différents intérêts sectoriels. Ce cas d'étude reflète le besoin de renforcer le système démocratique, avec une plus grande participation de la société civile.

Mots clés: groupes d'intérêt; pouvoir politique; taxes; boissons non alcoolisées.

\section{Cómo citar / How to cite this item:}

Díaz-García, J., Valencia-Agudelo, G., Carmona-Garcés, I. C. \& González-Zapata, L. I. (2020). Grupos de interés e impuesto al consumo de bebidas azucaradas en Colombia. Lecturas de Economía, 93, 155-187.

https://doi.org/10.17533/udea.le.n93a338783 


\title{
Grupos de interés e impuesto al consumo de bebidas azucaradas en Colombia
}

\author{
Juliana Díaz-García (1) ${ }^{\mathrm{a}}$, Germán Valencia-Agudelo $\mathbb{1}^{\mathrm{b}}$, Isabel Cristina \\ Carmona-Garcés $\mathbb{1}^{\mathrm{c}}$ y Laura Inés González-Zapata $\mathbb{1}^{\mathrm{d}}$
}

-Introducción. -I. Referentes conceptuales. -II. Metodología. -III. Resultados. -Discusión final. -Referencias.

Primera versión recibida el 11 de junio de 2019; versión final aceptada el 13 de abril de 2020

\section{Introducción}

En la actualidad los sistemas democráticos tienen un gran reto, dada la diversidad de actores involucrados en el proceso de elaboración y puesta en marcha de las políticas públicas. De allí que se requiera, por un lado, establecer métodos especiales de organización, delegación, especialización y dirección, que permitan a los órganos legislativo y ejecutivo emprender adecuadamente los diferentes momentos del ciclo de la política; por el otro,

a Juliana Díaz-García: profesora de cátedra de la Escuela de Nutrición y Dietética de la Universidad de Antioquia, Medellín, Colombia. Dirección electrónica: juliana.diaz@udea.edu.co https://orcid.org/0000-0002-5123-2413

b Germán Valencia-Agudelo: profesor titular del Instituto de Estudios Políticos de la Universidad de Antioquia e Integrante de los grupos de investigación Hegemonía, Guerras y Conflictos, y Microeconomía Aplicada de la Universidad de Antioquia, Medellín, Colombia. Dirección electrónica: german.valencia@udea.edu.co

https:// orcid.org/0000-0002-6412-6986

c Isabel Cristina Carmona-Garcés: profesora de la Escuela de Nutrición y Dietética de la Universidad de Antioquia e integrante del Grupo de Investigación Interdisciplinaria en Educación para la Salud y Educación Nutricional de la Universidad de Antioquia, Medellín, Colombia. Dirección electrónica: isabel.carmona@udea.edu.co

https://orcid.org/0000-0002-9757-7054

d Laura Inés González-Zapata: profesora titular de la Escuela de Nutrición y Dietética de la Universidad de Antioquia y Coordinadora del Grupo de Investigación en Determinantes Sociales y Económicos de la Situación de Salud y Nutrición, de la Universidad de Antioquia, Medellín, Colombia. Dirección electrónica: laura.gonzalez@udea.edu.co https://orcid.org/0000-0002-0345-3799 
se precisa controlar los conflictos del gran número de intereses que presionan libremente con sus demandas (Lindblüm, 1991, p. 86).

Los grupos de interés o Stakeholders son reconocidos como actores decisores de las políticas públicas (Saurugger, 2016; McKay \& Webb, 2007; Victor, 2007; Dusso, 2010). En estos se encuentran la empresa privada, los ciudadanos influyentes, las agrupaciones de trabajadores, los periodistas, los líderes de la opinión pública y las organizaciones no gubernamentales $(\mathrm{ONG})$, entre otros grupos de la sociedad civil (Lindblüm, 1991, p. 58). Los grupos de interés ponen en evidencia el cambio de paradigma de la gestión pública, en el cual la administración estructural funcionalista era el único organismo encargado de tomar decisiones y de poner en práctica las reglas formales, pero pasó a convertirse en un espacio de incidencia de un gran número de actores económicos, sociales, políticos y gubernamentales, con relaciones que pueden ser equitativas, simétricas, incluyentes o, por el contrario, dominantes, con base en su poder y estamento (Aguilar, 2015, p. 71).

En Colombia algunos grupos de interés influencian el sistema democrático. Uno de los casos que puso de manifiesto esta situación se evidenció en la pasada reforma tributaria presentada por el Gobierno nacional en 2016. En ella se propuso crear un impuesto saludable a las bebidas azucaradas, que permitiera desincentivar el consumo en la población colombiana. Esta propuesta surgió debido a que existe suficiente evidencia que soporta la magnitud de la problemática de sobrepeso y obesidad asociada al consumo de estas bebidas, y al riesgo de contraer enfermedades no transmisibles (OMS, 2015). La carga estimada de enfermedades mundiales, regionales y nacionales relacionadas con el consumo de bebidas azucaradas en 2010 (Singh et al., 2015), por ejemplo, evidenció que 184000 muertes al año se atribuyeron al consumo de bebidas azucaradas, con mayor prevalencia en países de medianos ingresos, como es el caso de México, que fue el país con mayor mortalidad asociada a este consumo. En Colombia se reportó un consumo de bebidas tipo gaseosa o refrescos por el 81,2\% de los habitantes, de los cuales se observó que casi 1 de cada 3 personas entre 14 y 30 años lo hace a diario (MPS, INS \& ICBF, 2010). 
Además, se justificó la creación del impuesto con la necesidad de generar ingresos fiscales para contribuir a financiar los gastos que el consumo de bebidas azucaradas acarrea para el sistema de salud pública (Ministerio de Hacienda y Crédito Público, 2016, p. 178; OMS, 2015). Esto debido a que los impuestos son el mecanismo más comúnmente utilizado por los Estados modernos para mitigar los costos de las externalidades negativas, es decir, de las acciones de carácter privado que afectan en forma negativa el bienestar de la sociedad (Ministerio de Hacienda y Crédito Público, 2016, p. 7; Stiglitz, 2000). Los debates giraron en torno a temas como el carácter regresivo o progresivo del impuesto y los efectos en la población de menores ingresos, que verían limitado su acceso a las bebidas azucaradas. Este último argumento se rebatía con la afirmación de los efectos beneficiosos que podría tener esta circunstancia en su salud, si se tiene en cuenta que la obesidad y la diabetes afectan con mayor frecuencia a las personas en condiciones de pobreza, y que el impuesto contribuiría a financiar el sistema de salud (Ministerio de Hacienda y Crédito Público, 2016, p. 178).

El impuesto nacional al consumo de bebidas azucaradas contempló una tarifa de 300 pesos por litro (entre 10 y 15 centavos de dólar) o su equivalente a bebidas gaseosas, bebidas a base de malta, bebidas tipo té o café, bebidas a base de fruta en cualquier concentración, refrescos y néctares de fruta, bebidas energizantes, bebidas deportivas, refrescos, aguas endulzadas y polvos que sirvan como base para preparar bebidas; esto tanto para las producidas nacionalmente como para las importadas y bajo la responsabilidad de los productores o importadores de estos productos (Congreso de la República de Colombia, 2016).

Alrededor de la propuesta surgieron distintas posturas de los actores y grupos de interés implicados, entre ellos el Congreso de la República, la industria de bebidas y, algunos otros miembros de la sociedad civil, como investigadores nacionales e internacionales, medios de comunicación y algunas ONG (García, Villar-Uribe \& Iunes, 2017, pp. 27-36). Esta medida tributaria no fue incluida por la Comisión Tercera de la Cámara de Representantes en el paquete de reforma tributaria entregado al Congreso de la República el 5 de diciembre de 2016, razón por la cual no pudo ser debatida en esta instancia. Lo anterior, marca un antecedente en la agenda de política 
Díaz-García, Valencia-Agudelo, Carmona-Garcés y González-Zapata: Grupos de interés...

pública en cuanto a impuestos saludables, considerando que Colombia ha sido el único país en el mundo que, presentada esta iniciativa, no la somete a consideración del Poder Legislativo.

Cabe decir que, después del debate de este impuesto saludable a las bebidas azucaradas, en la reforma tributaria aprobada en 2018, se incluyó un impuesto monofásico a las mismas; sin embargo, no tiene el objetivo de financiar los costos de las externalidades negativas ni de desestimular el consumo por razones de salud, como es recomendado por los expertos del tema en el mundo, dada su relación estrecha con el sobrepeso, la obesidad, la diabetes, la caries dental, entre otros (Nestle, 2015, pp. 53-77).

Este artículo aborda los sucesos alrededor de la propuesta de impuesto saludable a las bebidas azucaradas en Colombia en el 2016, como un ejemplo que evidencia con claridad el poder de grupos de interés en las decisiones públicas del país, y en este caso expresado por la capacidad de intervención y decisión de la industria de bebidas azucaradas. Para abordar este tema, se da respuesta a las siguientes preguntas: ¿Cómo fue el proceso decisorio en torno a la propuesta de impuesto al consumo de bebidas azucaradas? y ¿cuál fue el papel de los diferentes grupos de interés en ese proceso?

\section{Referentes conceptuales}

\section{A. La formación de agenda y la definición del problema público}

El objeto de análisis de este trabajo se inscribe, de acuerdo con Aguilar (2015), en las dos primeras fases del ciclo de política pública: la formación de agenda y la definición del problema público, las cuales son "operaciones intelectuales y políticas que la decisión de la política requiere llevar a cabo para ser pública, real y eficaz” (p. 59). Estas fases constituyen una decisión en sí misma porque los diferentes actores involucrados en el proceso determinan si una situación es un problema público o no, en qué consiste el problema, sus causas y consecuencias, cuáles deberían ser los mecanismos de actuación, qué instrumentos de intervención se requerirían, qué recursos son necesarios y cuáles son los resultados esperados, entre otros aspectos. 
En las discusiones recientes sobre estas dos etapas del ciclo de las políticas públicas y la manera de resolver todos los asuntos por los que allí se indagan, se encuentran dos enfoques analíticos dominantes: el pluralismo democrático y la elección pública (Public Choice). El primer enfoque, proveniente de la ciencia política, defiende la idea de una fragmentación de la sociedad y del Estado; presenta a ambos actores como compuestos por grupos diversos, con intereses dispares y que disponen de un amplio abanico de recursos para influir en el sistema de gobierno (o Estado), que a la vez también es diverso y conflictivo (Medina, 2009). Para el pluralismo, el Estado lo integra un conjunto de instituciones, tales como los poderes Ejecutivo, Legislativo y Judicial; estos actores reflejan las demandas de la sociedad y se ven constreñidos por el poder compensador de quienes representan (sociedad civil y otras organizaciones) (Smith, 1997).

El aporte de este enfoque a la relación entre políticas públicas y grupos de interés está en el énfasis en el estudio de las motivaciones de los actores y el entendimiento de las lógicas subyacentes, y además en la definición de los comportamientos dados en las arenas volátiles del ejercicio del gobierno (Vargas, 2008). También el enfoque permite resaltar el papel que tienen los empresarios, como grupo de interés, en el sistema democrático; muestra su privilegio en términos de posesión de recursos e influencia sobre las decisiones del Estado (Valencia, 2005). Finalmente, posibilita analizar las interacciones entre gobierno y grupos de interés, ya sea que se mire al gobierno como grupo de interés (ministerios, funcionarios públicos, legisladores, etc.) o en su interacción con agentes externos (empresarios, sindicatos, asociaciones de consumidores, etc.). En definitiva, el pluralismo democrático es útil para poner la mira en los grupos de interés, identificando sus motivaciones, valores y forma de operar para ejercer presión en la toma de decisiones del gobierno.

El segundo enfoque, proveniente de la economía, es defendido por la teoría de la elección racional. Presenta la decisión de política pública como una actividad racional, en la cual los agentes tienen que elegir, entre diversas opciones, aquella que sea más conveniente o mayor bienestar les dé (individual o colectivamente) (Vidal, 2008). Según este enfoque, el Estado como representante de la sociedad debería intervenir en los diversos 
Díaz-García, Valencia-Agudelo, Carmona-Garcés y González-Zapata: Grupos de interés...

mercados, con el fin de minimizar los efectos secundarios (externalidades negativas) que tiene el consumo de un bien o servicio, y proponer a la industria unos acuerdos de riesgo compartido. Esta visión muestra que los agentes, sean individuales o grupales, deben decidir sobre cuál es la mejor forma posible de alcanzar sus metas teniendo en cuenta el comportamiento racional y estratégico, y las interacciones ambientales.

Dado el poder analítico que tienen ambos enfoques en el análisis de las políticas públicas y los grupos de interés, es conveniente utilizarlos conjuntamente. Se retoma de la elección racional la idea maximizadora de utilidad, y del pluralismo democrático la manera como analiza los grupos de interés y como estos utilizan los instrumentos de poder que tienen a su alcance para ejercer incidencia y lograr sus objetivos. Ambos enfoques ayudan a analizar la toma de decisiones públicas como resultado de una interacción de numerosas fuerzas y pasiones, sujetos a influencias de diversos órdenes y siempre relacionados en las luchas por el poder, el ejercicio y el control (Vargas, 2008).

\section{B. Incidencia y poder en las políticas públicas}

Durante el ciclo de las políticas, la formación de agenda es una etapa sumamente frágil, la cual requiere de la participación de los tomadores de decisiones y de una acción colectiva de la sociedad civil, en cabeza de cualquier organización que la represente (ONG, organizaciones de base o movimientos sociales, entre otras). Estos actores son claves en la dinámica social de los sistemas democráticos, deben trabajar para fortalecer los mecanismos de incidencia en la agenda y velar por la permanencia y priorización de sus intereses (Valencia, 2012, p. 480). No obstante, no son los únicos que se movilizan para penetrar la agenda del gobierno y lograr imponer sus preferencias, otros grupos de interés económicos, privados y políticos, entre otros, también intervienen. Ello evidencia que las políticas públicas son un juego político resultado de la incidencia de múltiples actores que luchan para lograr su inclusión en la toma de decisiones políticas.

En este sentido, vale la pena definir el término incidencia en las políticas públicas como las "actividades confrontativas y cooperativas que implican 
interactuar con el gobierno y otras instituciones públicas" (Tapia et al., 2010, p. 13). Sin embargo, para que sea efectiva, es decir, para que llegue a permear las decisiones públicas, Valencia (2012) señala algunos retos que tienen los actores, especialmente la sociedad civil, como "aumentar la legitimidad, fortalecerse en conocimientos e información, aumentar los recursos para tener mayor autonomía frente al Estado, utilizar mejor sus instrumentos de poder para decidir e incidir" (p. 488), entre otros.

Según Bachrach y Baratz (1963), el poder y la influencia pueden y deben distinguirse. Sin embargo, la línea entre ellos es difícil de trazar. Su dificultad radica en el hecho de que los dos, en ocasiones, se refuerzan mutuamente; esto es, el poder genera frecuentemente influencia y viceversa. La amenaza de sanciones es lo que hace diferente al poder de la influencia. Para que una relación de poder exista debe cumplir tres condiciones, a saber:

Primero debe haber un conflicto de intereses o de valores entre dos o más personas o grupos. Segundo, $A$ accede realmente a los deseos de $B[\ldots]$, y tercero, una relación de poder es posible sólo si una de las partes puede amenazar al invocar sanciones: poder es el proceso de afectar las políticas de otros con la ayuda de severas sanciones (...amenazando), por no estar conforme con las políticas deseadas (Bachrach \& Baratz, 1963, p. 83).

En el caso particular de este trabajo, poder e influencia se abordaron como dos variables independientes para determinar cómo estas se reforzaron mutuamente en la toma de decisiones.

\section{Los grupos de interés en las políticas públicas}

Algunos de los actores reconocidos por su incidencia y poder en la política pública y de particular relevancia en este trabajo, son los grupos de interés. Estos son definidos por David Truman (1951), quien introdujo el concepto en 1951 por primera vez, como "cualquier grupo que, sobre la base de una o más actitudes compartidas, hace ciertas reivindicaciones sobre otros grupos en la sociedad para el establecimiento, mantenimiento o mejora de formas 
Díaz-García, Valencia-Agudelo, Carmona-Garcés y González-Zapata: Grupos de interés...

de comportamiento” (p. 246). Por otra parte, nociones más contemporáneas entienden las actividades de los grupos de interés como:

Todas las interacciones a través de las cuales los individuos y grupos privados sin autoridad gubernamental pretenden influir en las políticas públicas, junto con las interacciones e influencias de los funcionarios del gobierno que van más allá del uso directo de su autoridad (Lindblüm, 1991, p. 110).

Saurugger (2016) sugiere incluir entre los grupos de interés, además de la empresa privada, los ciudadanos influyentes, las agrupaciones de trabajadores, los periodistas y otros líderes de la opinión pública, las ONG y la sociedad civil, y las empresas y servicios del Estado, dado que "el Estado es un receptor de demandas provenientes de grupos de interés, al tiempo que sus servicios administrativos o políticos pueden ellos mismos transformarse en grupos de interés" (p. 331).

Con respecto a considerar o no a las personas con cargos públicos como grupos de interés, Lindblüm (1991, p. 109) señala que la línea divisoria es estrecha. En el caso de un miembro del Congreso que realiza una votación a favor o en contra como ejercicio de su rol para el Estado, no estaría ejerciendo una actividad de grupo de interés, mientras que, si intenta persuadir a un funcionario para influir en una política o con un profesional del lobby intenta influir a otros miembros del Congreso, es evidente que está haciéndolo. En este sentido y de acuerdo con la hipótesis del presente trabajo, los actores gubernamentales serán considerados como grupos de interés.

De acuerdo con Chávez (2006),

El Poder Legislativo o parlamento es sin duda la institución más importante del Estado moderno. Diversos autores se han ocupado en definirlo; la palabra parlamento hace referencia a la institución representativa de un Estado integrada por los propios ciudadanos mediante procesos electorales abiertos, que participan de manera insustituible en la formación de la voluntad general del Estado mediante la aprobación de leyes y normas financieras estatales, y controla en mayor o menor grado la actividad del gobierno (p. 94). 
Se habla de que la principal función de los grupos de interés es la vigilancia; sin embargo, en función de sus actividades particulares ejercerán influencia para modificar una situación, incluso si va en contravía del bienestar común de la población. Algunas fuentes de incidencia e instrumentos de poder de los grupos de interés son: la persuasión, la influencia a través de la reglamentación, la entrega del voto y el apoyo a las campañas electorales (Lindblüm, 1991, pp. 113-116).

También existen otros instrumentos de poder disponibles, como la capacidad analítica, la capacidad organizativa y el dinero, los cuales generan desigualdad en las políticas debido a que están distribuidas inequitativamente y provocan que los diferentes grupos de interés no puedan ejercer una misma incidencia. En este sentido, Lindblüm (1991, p. 121) señala que los grupos empresariales tienen la ventaja frente a otros grupos de disponer de finanzas y de una mejor organización, así como de poder de negociación en términos de impacto al sector económico, laboral, social, ambiental, entre otros; por tanto, su capacidad de persuasión y presión es comparativamente mayor.

Por último, es necesario hablar de la noción de decisión o, para este caso particular, de la no decisión, de acuerdo con Bachrach y Baratz (1963), al decidir no considerar el impuesto al consumo de bebidas azucaradas propuesto como una manera de resolver el problema público de sobrepeso y obesidad. El concepto decisión en nuestro objeto de estudio está dado por la decisión pública, y esta es entendida como "el producto de un arbitraje constante entre la factibilidad política y la racionalidad económica o técnica y el resultado de la articulación entre policy y politics, entre la acción pública y sus públicos" (Halpern, 2016, p. 196). El proceso decisional no es lineal, se elabora bajo restricciones como información incompleta, reglas informales, entre otras. Algunos autores lo definen como un proceso caótico que se da en función de la movilización de los distintos intereses sectoriales y consiste en un enjeu de poder (intereses políticos en juego) (Halpern, 2016), que se ve reflejado en situaciones como la presentada en este trabajo. 
Díaz-García, Valencia-Agudelo, Carmona-Garcés y González-Zapata: Grupos de interés...

\section{Metodología}

Este estudio está basado en el análisis de interesados o Stakeholder Analysis, el cual centra la atención en los diferentes actores intervinientes en un determinado proceso de política pública y el rol que estos desempeñan en torno a la misma. Para este análisis, la metodología empleada se dividió en dos partes: una que aborda el proceso decisorio del impuesto al consumo de bebidas azucaradas y otra en torno al papel de los diferentes grupos de interés en ese proceso.

\section{A. El proceso decisorio}

Para el análisis del proceso decisorio, así como de las diferentes posturas y roles asumidos por los diferentes actores, se realizó una revisión documental, que inicia en diciembre de 2015, momento en el cual comenzó a gestarse la propuesta del impuesto a las bebidas azucaradas en cabeza del Ministerio de Salud y Protección Social —MinSalud_- y finaliza el 5 de diciembre de 2016, cuando se toma la decisión de no incluir el impuesto en el paquete de reformas tributarias para ser sometido a consideración del Legislativo. Se incluyeron en el análisis las diferentes publicaciones realizadas por los grupos de interés en sus páginas oficiales, comunicados, artículos de prensa y publicaciones en redes sociales, principalmente de Twitter por ser la plataforma de distribución de información, posiciones o noticias de facto más usada.

Como grupos de interés para este trabajo se consideraron los siguientes actores clave por su representación de los diferentes sectores involucrados o su incidencia en el proceso decisorio: Congreso de la República, Asociación de Industriales de Colombia (ANDI), Federación Nacional de Comerciantes (Fenalco), MinSalud, Ministerio de Hacienda, medios de comunicación, Superintendencia de Industria y Comercio (SIC), algunas ONG y el sector académico. Adicionalmente, se realizaron seis entrevistas estructuradas a: un representante de MMinSalud, un congresista, un representante de Fenalco, un periodista y columnista del periódico El Colombiano con publicaciones respecto al tema, un representante de la ONG Educar Consumidores y un académico de la Universidad Javeriana de Bogotá con amplia trayectoria 
investigativa y participación activa alrededor de la propuesta al consumo de bebidas azucaradas, quienes aceptaron participar como voluntarios y voluntarias en la investigación, y diligenciaron el consentimiento informado para el uso de la información.

En la entrevista se indaga por aspectos generales, como la consideración de las enfermedades crónicas no transmisibles, el sobrepeso y la obesidad como un problema de salud pública, sus factores de riesgo, las medidas que deben tener el Estado, la industria y el individuo para afrontarlas y la apreciación acerca de los efectos que se les atribuyen a los impuestos saludables. Tambien se preguntó por la percepción acerca de la propuesta y del proceso decisorio del impuesto al consumo de bebidas azucaradas y si se observan restricciones para la toma de decisiones y, finalmente, se indaga por el rol como persona o representante de alguna institución durante el proceso.

\section{B. El papel de los diferentes grupos de interés}

Con el propósito de determinar las relaciones entre los diferentes grupos de interés y su incidencia y poder, se realizó una revisión bibliográfica de las diferentes metodologías empleadas y no se encontró un consenso metodológico para este análisis. El presente trabajo propone unas escalas de valoración para ello y recoge parte de la metodología empleada en Mapa de actores para la construcción de una política pública de trabajo decente en Medellín (Centro de Análisis Político Universidad EAFIT, 2012), la cual retoma a su vez instrumentos del Banco Mundial para el análisis de interesados (Stakeholder Analysis).

El procedimiento realizado fue el siguiente: a los actores entrevistados se les solicitó una valoración de 1 a 5 de las variables relaciones, incidencia y poder, sobre la decisión de no establecer la medida fiscal, siendo 1 ninguno y 5 mucho; un ejercicio igual se realizó para las mismas variables teniendo en cuenta lo que para el entrevistado sería un escenario ideal. Con estos elementos se construyeron los mapas de actores, de acuerdo con la metodología planteada para el análisis de interesados; "esta es una metodología sistemática que usa datos cualitativos para determinar los 
Díaz-García, Valencia-Agudelo, Carmona-Garcés y González-Zapata: Grupos de interés...

intereses e incidencia de diferentes grupos en relación con una reforma" (Banco Mundial, 2003, p. 17).

Si bien es cierto que el método usa datos cualitativos a partir de los instrumentos mencionados, para efectos de este estudio se establecieron promedios de los niveles de incidencia y poder según las calificaciones dadas por los entrevistados. Se ilustró en un gráfico de dispersión el cruce de las variables, y las líneas se trazaron de acuerdo con los promedios de incidencia en el eje vertical, y de poder en el eje horizontal, para reflejar la ubicación del grupo de interés en la toma de decisiones con respecto al impuesto. Aquellos actores más relevantes en el proceso se encontraron en el cuadrante I, donde coinciden los valores más altos de las variables analizadas (Tabla 1). Finalmente, el análisis se realizó mediante la transcripción de las entrevistas y se usó el software SPSS versión 23 (SPSS Inc., Chicago, EE. UU.) para la elaboración de gráficos de barras y de dispersión para los mapas de actores.

Tabla 1. Esquema para la elaboración de los mapas de actores

\begin{tabular}{|c|c|c|c|}
\hline \multicolumn{2}{|c|}{ Escenario real en que se dio el proceso } & \multicolumn{2}{|c|}{ Escenario ideal } \\
\hline Cuadrante II & Cuadrante I & Cuadrante II & Cuadrante I \\
\hline Incidencia $^{-}$, Poder $^{+}$ & Incidencia $^{+}$, Poder $^{+}$ & Incidencia $^{-}$, Poder $^{+}$ & Incidencia $^{+}$, Poder $^{+}$ \\
\hline Cuadrante III & Cuadrante IV & Cuadrante III & Cuadrante IV \\
\hline Incidencia $^{-}$, Poder ${ }^{-}$ & Incidencia $^{+}$, Poder ${ }^{-}$ & Incidencia $^{-}$, Poder ${ }^{-}$ & Incidencia $^{+}$, Poder ${ }^{-}$ \\
\hline
\end{tabular}

Fuente: construcción a partir de la metodología empleada en Mapa de actores para la construcción de una política pública de trabajo decente en Medellin (2012, p. 174).

\section{Resultados}

\section{A. Proceso decisorio en torno a la propuesta de impuesto al consumo de bebidas azucaradas}

La propuesta comenzó a gestarse desde diciembre de 2015 en cabeza de MinSalud, con participación de la sociedad civil, en particular de las ONG Educar Consumidores y Dejusticia. Los antecedentes de la propuesta parten de reconocer la problemática de salud en la población colombiana asociada a la ingesta diaria de bebidas azucaradas y de las recomendaciones de diferentes 
investigaciones e instancias mundiales para reducir las tendencias actuales de producción en el país — crecimiento del $40 \%$ en el mercado de bebidas azucaradas entre 2009 y 2014 (Bustamante, 2014) — mediante la grabación de impuestos para su comercialización (OMS, 2015; Moodie et al., 2013; Popkin \& Hawkes, 2015; OPS, 2015).

Como una medida para anticiparse a la posible reglamentación, las empresas de bebidas no alcohólicas (Postobón, Pepsi Co, Coca Cola, Coca Cola FEMSA, Bavaria, Red Bull, AJE y Monster Energy), lideradas por la Cámara de la Industria de Bebidas de la Asociación Nacional de Empresarios de Colombia (ANDI), el 19 de mayo firmaron un pacto en el que se comprometieron a lo siguiente:
A junio de 2017 implementar un rotulado frontal en las bebidas para dar mayor información al consumidor, no dirigir publicidad a niños menores de 12 años, promover los estilos de vida activos y saludables, innovar y ampliar el portafolio, y no comercializar bebidas azucaradas en colegios y escuelas del país, [...] con el propósito de influenciar de manera positiva en los hábitos de consumo de los colombianos (ANDI, 2016, pp. 1-2).

Por su parte, investigadores y asociaciones iniciaron en este lapso el debate de la pertinencia del impuesto, pero solo hasta junio de 2016 el ministro de Salud lo consideró en la agenda pública y lo defendió para que fuera incluido en la reforma tributaria. A partir de allí comenzó la fase predecisional de la que se habló en el marco teórico, constituida por la formación de agenda y la definición del problema público. La Figura 1 describe los principales acontecimientos de esta fase hasta la no radicación del impuesto en el paquete de la reforma tributaria a ser debatida por el Congreso. Se elaboró con base en revisión bibliográfica, búsqueda en medios de comunicación, páginas web institucionales y diálogo con los grupos de interés entrevistados.

En las entrevistas a los grupos de interés, se indaga por la valoración de la propuesta como tal y del proceso seguido durante el periodo de tiempo señalado. Con respecto al diseño, se observa consistencia entre los actores que apoyaron o que no apoyaron el impuesto. Así, MinSalud menciona 
Díaz-García, Valencia-Agudelo, Carmona-Garcés y González-Zapata: Grupos de interés...

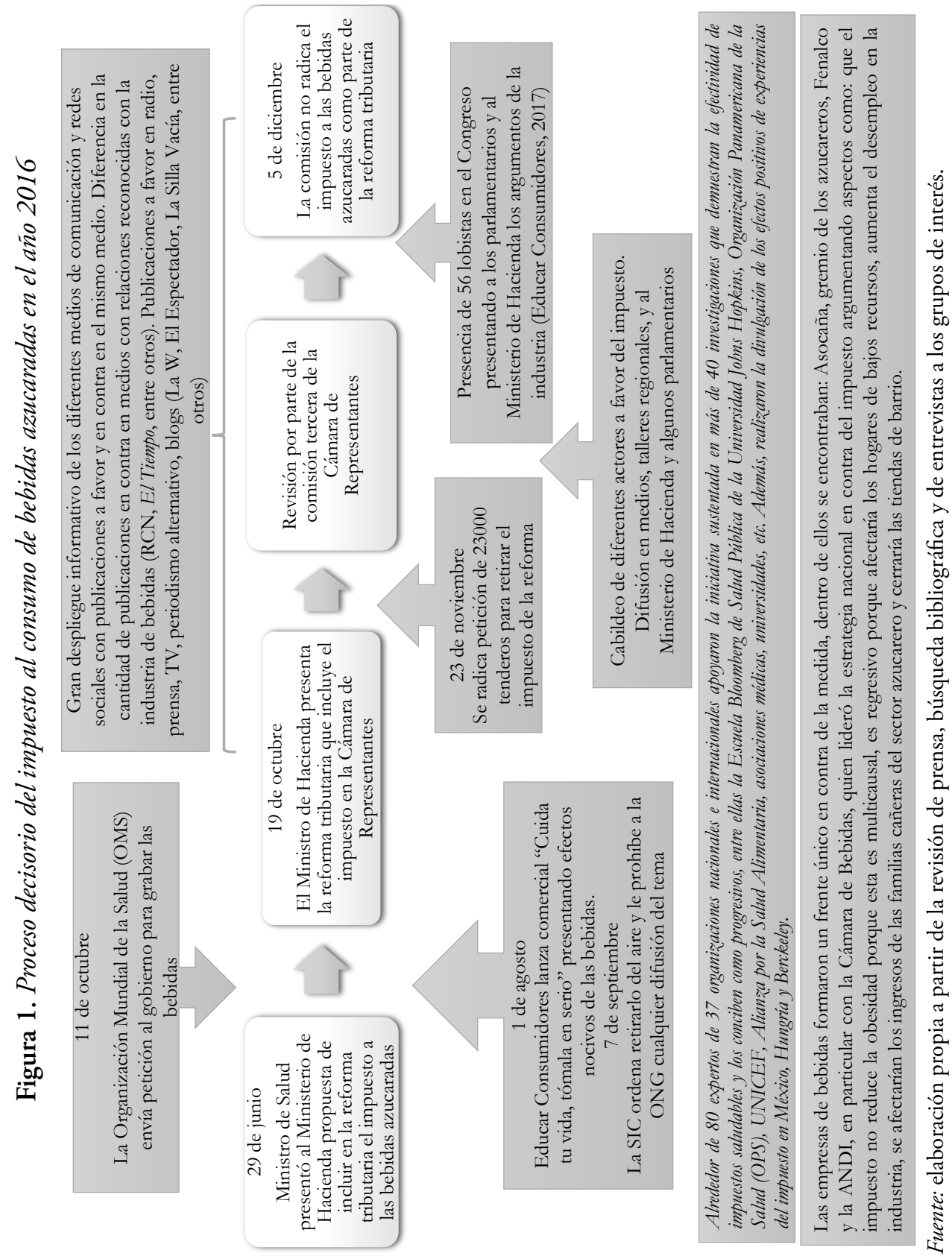


que "lo que está escrito lo tenemos tan revisado, tan estudiado, que lo único que mejoraría es la comunicación y cómo llegar a la gente y que lo entienda; sin duda visibilizar que no son acciones unitarias, sino integradas" (representante MinSalud, comunicación personal, 21 de mayo de 2017). Pese a haber participado en la construcción de la estrategia, un representante de la ONG Educar Consumidores considera que hay aspectos que se pueden mejorar:

Un impuesto al valor ( $\%$ al precio final del producto) y no un impuesto específico (300 pesos por litro en este caso), dado que el problema no es solo el azúcar de la bebida, sino el conjunto como tal de los demás ingredientes. Y para que tenga efectos reales debe quedar lo más cerca del consumidor, porque si no, la industria lo absorbe y lo disfraza, como lo sucedido con los empaques pequeños y la plataforma de las light (comunicación personal, 26 de abril de 2017).

Por su parte, el representante del gremio de los comerciantes y quien comparte la visión de la industria menciona:

Lo sucedido con el impuesto evidencia cómo por llenar un déficit fiscal, $[\ldots]$ por falta de racionalidad en el manejo de la hacienda pública se buscan impuestos indiscriminadamente sin fundamentos técnicos y científicos, además porque hicimos un estudio en el caso de las bebidas endulzadas y muchos países tuvieron que regresarlo cuando también lo establecieron con el pretexto de favorecer la salud de clientes y consumidores (comunicación personal, $17 \mathrm{de}$ mayo de 2017).

En la valoración del proceso de toma de decisiones, el representante de Fenalco percibió:

No fue una propuesta socializada con la industria y el pequeño comercio representado en el pequeño tendero. [...] Por nuestra parte, nosotros llevamos la voz de los tenderos y de los clientes 
Díaz-García, Valencia-Agudelo, Carmona-Garcés y González-Zapata: Grupos de interés...

y consumidores y aportamos informaciones valiosas al Ministerio de Hacienda y al Congreso de la República sobre que estas bebidas representan alrededor del $30 \%$ de los ingresos del pequeño comercio [...], que millones de personas de estratos 1,2 y 3 tienen en su dieta permanente en su desayuno, almuerzo y comida una de estas bebidas endulzadas, entonces es una afectación para la dieta (comunicación personal, 17 de mayo de 2017).

Relacionado con estos argumentos, el congresista entrevistado percibe que MinSalud debió adelantarse a responder esas críticas y a expresar de manera contundente, con ejemplos de vida, los efectos positivos que podría tener la implementación del impuesto:

Eso fue escalando y ahí arranca todo el tema de la postverdad. [...] Yo creo que en ese proceso estuvo muy ausente el papel de los centros de pensamiento, académicos, donde se pudiera debatir y oír argumentos por fuera de la industria. El papel de ellos sí fue muy fuerte, tenía lobistas, personas que se van para allá donde el congresista y le llevan una carpeta a uno con sus argumentos, incluso se van con sus expertos y le piden a uno que los escuche y [explican] por qué el tema en México no funcionó. Entonces se vio que ellos se movieron mucho, es como esa institución informal que funciona muy eficientemente [...]. No puedo decir y no me consta que haya habido corrupción u ofrecimiento de dinero para votar o no (comunicación personal, 19 de mayo de 2017).

El representante de MinSalud percibió debilidades en el proceso al considerar que:

Nuestro punto débil fue que [se vio] la estrategia como si fuera la única con la que enfrentábamos el problema y que pretendía solo buscar más dinero para el sistema de salud. Cuando nos dimos cuenta que esa era la percepción que se estaba teniendo, nuestra reacción fue un poquito lenta [...]. Hay un actor que es invisible y es cuando hay desinformación, y las redes sociales, así 
como sirven para difuminar información, también nos sirven para destruir (representante de MinSalud, comunicación personal, 21 de mayo de 2017).

Por último, en la evaluación del proceso los representantes de las ONG y el sector académico mencionan que fue una ganancia poner el impuesto en la agenda pública, así no se sometiera a consideración del Congreso en pleno. Sin embargo, piensan que el proceso en general no fue apropiado; era necesario que se tratara como una decisión de país, es decir, que el mismo presidente tuviera la bandera, no que fuera una pelea del ministro, aunque le reconocen su gran liderazgo en el tema. Al respecto, Educar Consumidores indica que:

Una debilidad del proceso es que tuviera en contra al ministro de Hacienda, pero era claro que si para que le aprobaran la reforma tributaria tenía que sacrificar algo, el impuesto a sacrificar era el de las bebidas, ni siquiera el de tabaco. La industria tiene sus brazos dentro del Ministerio de Hacienda, coaptó completamente también el escenario del Congreso, especialmente la Comisión Tercera, quien convocó escenarios públicos para escuchar a la sociedad civil frente a los impuestos, pero para ellos la sociedad civil era la ANDI, Fenalco, los azucareros y nosotros, Educar Consumidores, y por ejemplo la Javeriana en algunos momentos, pero nos tocó meternos a las malas (comunicación personal, 26 de abril de 2017).

\section{B. Consideraciones de los grupos de interés entrevistados acerca de los efectos que se les atribuyen a los impuestos saludables}

De manera general, los actores entrevistados reconocen la problemática nacional de enfermedades crónicas no transmisibles (ECNT), sobrepeso y obesidad como un problema de salud pública muy grave en el país y acuden a citar investigaciones que lo sustentan, como la valoración de la Organización Mundial de la Salud (OMS) como epidemia mundial. Entre los factores de riesgo para la aparición mencionan la relación con factores genéticos, pero con más influencia de factores contextuales como pobreza, 
Díaz-García, Valencia-Agudelo, Carmona-Garcés y González-Zapata: Grupos de interés...

estilos de vida poco saludables, sedentarismo, aumento en el consumo de calorías, hábitos alimentarios inadecuados, en particular consumo de productos ultraprocesados y bebidas azucaradas.

La indagación acerca de las medidas que deben implementarse para frenar y revertir la problemática se realizó con el propósito de establecer si consideran los impuestos saludables como un instrumento útil en su resolución. Se encontró que los representantes de Fenalco y los miembros del Congreso de la República opinan que el Estado debe cumplir un rol preventivo enfocado en la educación para crear hábitos saludables en los individuos y para que puedan tomar mejores elecciones, más que crear un marco normativo y obligatorio con medidas tributarias. Conciben un deber ser para la industria basado en la oferta y demanda, aludiendo a un consumo responsable de la población.

Por su parte, los demás Stakeholders consideran necesario que el Estado, además de crear entornos saludables, acompañe estas estrategias con un marco regulatorio para el mercadeo principalmente dirigido a niños, impuestos para limitar el consumo de productos pocos saludables y regulación de los entornos alimentarios escolares, entre otros. Al respecto, el actor del sector académico menciona que "estas políticas públicas deben ser legitimadas por la sociedad civil, ya hemos aprendido que las políticas prescriptivas desde el Estado no funcionan y tiene que haber un acuerdo social sobre el tema" (comunicación personal, 24 de mayo de 2017).

Pese a la coincidencia en el rol del Estado, se observan diferencias en el que hacer para enfrentar la problemática por parte de la industria y de los individuos. Los actores del sector académico y las ONG consideran que el rol regulatorio del Estado deber ser tan claro y normativo que debe determinar el tipo de producción y la oferta a la población; por lo tanto, no está en manos de la industria la solución. Por su parte, MinSalud y los medios de comunicación mencionan la necesidad de que la industria haga modificaciones en su portafolio y etiquetado, con miras a mejorar la oferta y brindar información veraz en sus empaques y su publicidad. 


\section{El papel de los diferentes grupos de interés en el proceso decisorio}

Finalmente, se presentan los resultados de las relaciones, así como su incidencia y poder de los diferentes grupos de interés. De acuerdo con las Figuras 2 y 3 , es posible analizar la calificación que los actores entrevistados le otorgan a cada uno de los grupos de interés considerados relevantes en el proceso. Se evidencia la mayor incidencia para los representantes de la industria, lo cual coincide con el grado de poder asignado para este mismo actor. El otro actor fundamental en las decisiones, según lo esbozado en párrafos anteriores, es el Ministerio de Hacienda, seguido con el mismo nivel de incidencia de los medios de comunicación. Con respecto a la Superintendencia de Industria y Comercio, pese a que no tiene tanto poder en la toma de decisiones, se refleja una incidencia igual a la de Fenalco, debido al antecedente de la prohibición del comercial de la ONG Educar Consumidores "Cuida tu vida, tómala en serio" que presentaba los efectos nocivos de las bebidas, y del veto a toda forma de publicidad en este sentido. La baja incidencia y poder de MinSalud, las ONG y el sector académico concuerdan con la decisión final de la no aprobación del impuesto.

Figura 2. Incidencia según grupo de interés

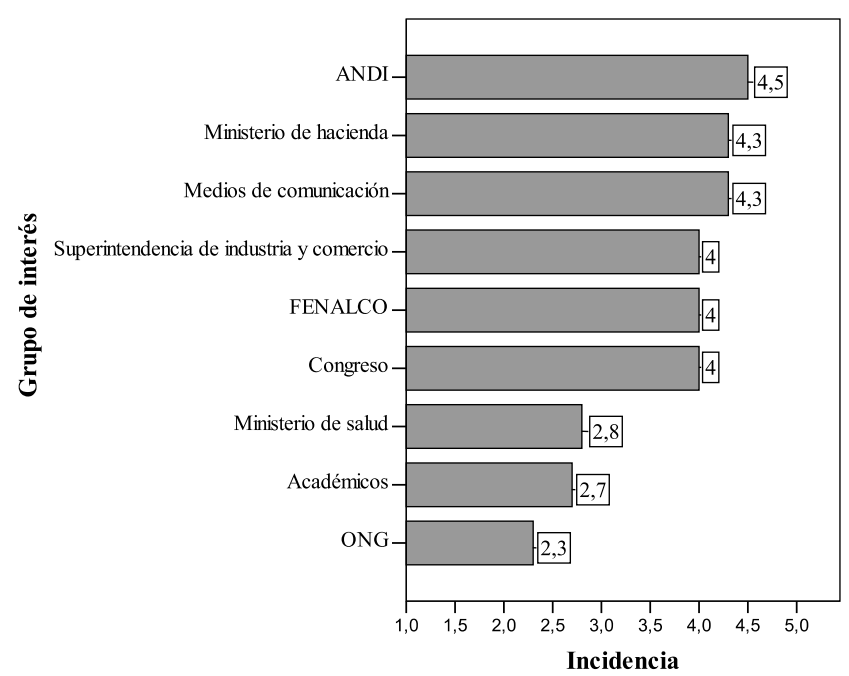

Fuente: elaboración propia. 
Díaz-García, Valencia-Agudelo, Carmona-Garcés y González-Zapata: Grupos de interés...

Figura 3. Poder según grupo de interés

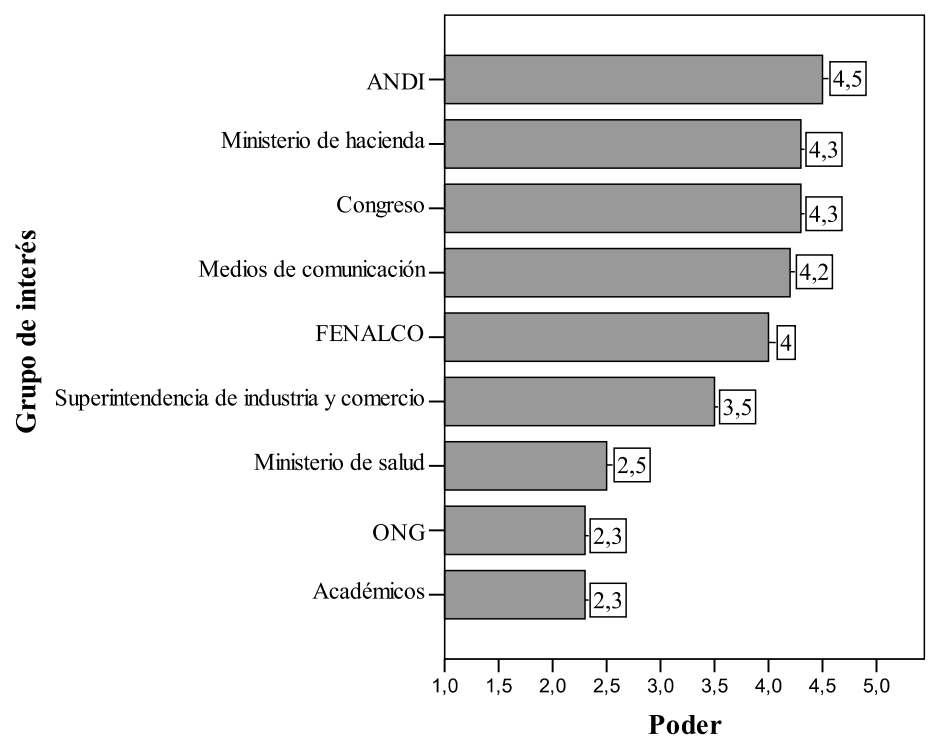

Fuente: elaboración propia.

La variable de relaciones entre actores solo puede ser representada para las relaciones de los seis grupos de interés entrevistados, considerados como involucrados o con incidencia en el proceso decisorio. Se puede apreciar en la Figura 4 que en general no existen relaciones muy estrechas entre los grupos de interés; no obstante, la ONG Educar Consumidores tiene el grado más alto de relaciones entre los grupos de interés, seguido muy de cerca por Fenalco. Esto puede tener explicación desde el ejercicio de cabildeo y lobby realizado por este actor con los demás grupos de interés.

Como se mencionó en la metodología, a partir de las variables incidencia y poder se construyeron los mapas de actores, con el propósito de discriminar cuáles grupos de interés fueron determinantes para no establecer la medida fiscal. Además, al haber indagado por el deber ser de la incidencia y el poder en las decisiones públicas de los mismos, se sugiere un mejor escenario para la toma de decisiones. Para cada caso, los actores que se ubiquen en el cuadrante superior derecho de cada mapa deberán ser analizados como actores relevantes en el proceso decisorio, mientras que aquellos ubicados 
en el cuadrante inferior izquierdo, como quienes menos poder e incidencia representaron en el proceso.

Figura 4. Relaciones entre los grupos de interés

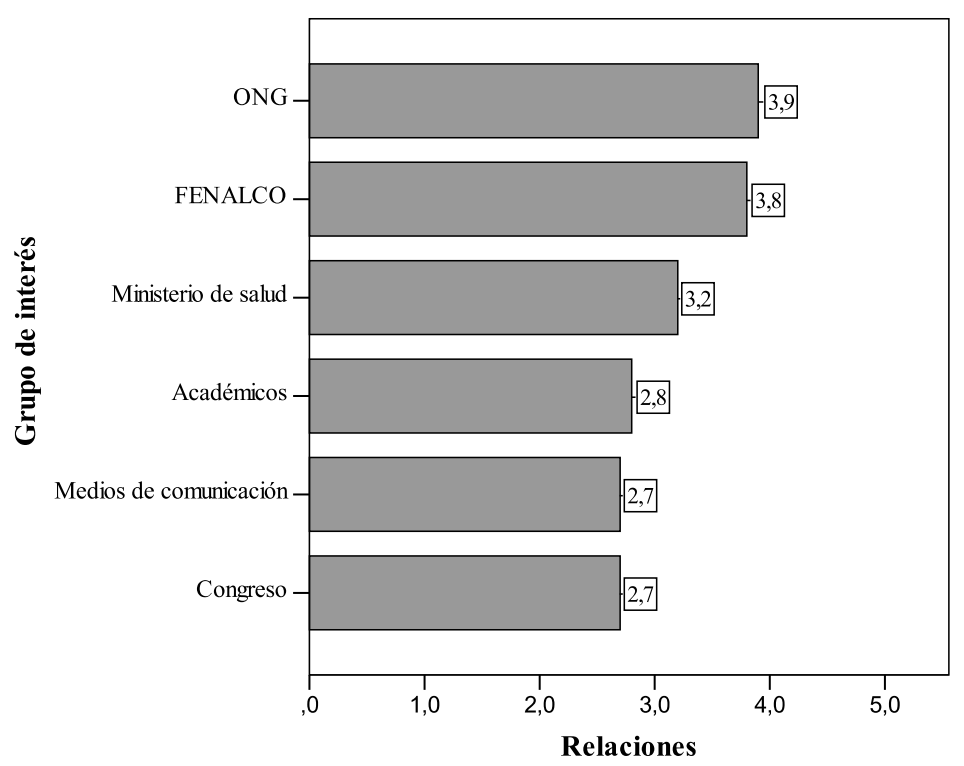

Fuente: elaboración propia.

La Figura 5 muestra el mapa de actores. Se puede apreciar el modesto poder e incidencia de MinSalud, el sector académico y las ONG, lo cual sugiere que no fueron determinantes en la toma de decisiones con respecto al impuesto. En el cuadrante superior se ubica el Congreso, con mayor poder que incidencia, mientras que se evidencia que el actor más influyente en la no aprobación del impuesto fue la ANDI, en representación de la industria y del sector azucarero. Sumado a esto se encuentra el rol para nada despreciable que tuvo Fenalco, en representación de los comerciantes.

Esta situación cambia drásticamente al plantearse a los entrevistados el escenario ideal de toma de decisiones con respecto al impuesto. El rol de la SIC, la ANDI y Fenalco prácticamente se invierte. Al respecto, los actores entrevistados consideran que la ANDI y Fenalco no deberían ser juez y parte 
Díaz-García, Valencia-Agudelo, Carmona-Garcés y González-Zapata: Grupos de interés...

en el caso; su poder e incidencia debe ser limitado. El rol de los medios de comunicación, si bien continúa siendo importante en términos de incidencia, su poder debe ser restringido en aras de cumplir su función de comunicar e informar con veracidad. Se evidencia una fuerte reivindicación de la sociedad civil, en cabeza de las ONG y el sector académico. Y se le delega al Congreso un poder más contundente como legislativo y tomador de decisiones, además se observa que tanto MinSalud como el de Hacienda deberían tener un importante rol en la toma de decisiones (Figura 6).

Figura 5. Mapa de actores, incidencia y poder en el escenario de la decisión

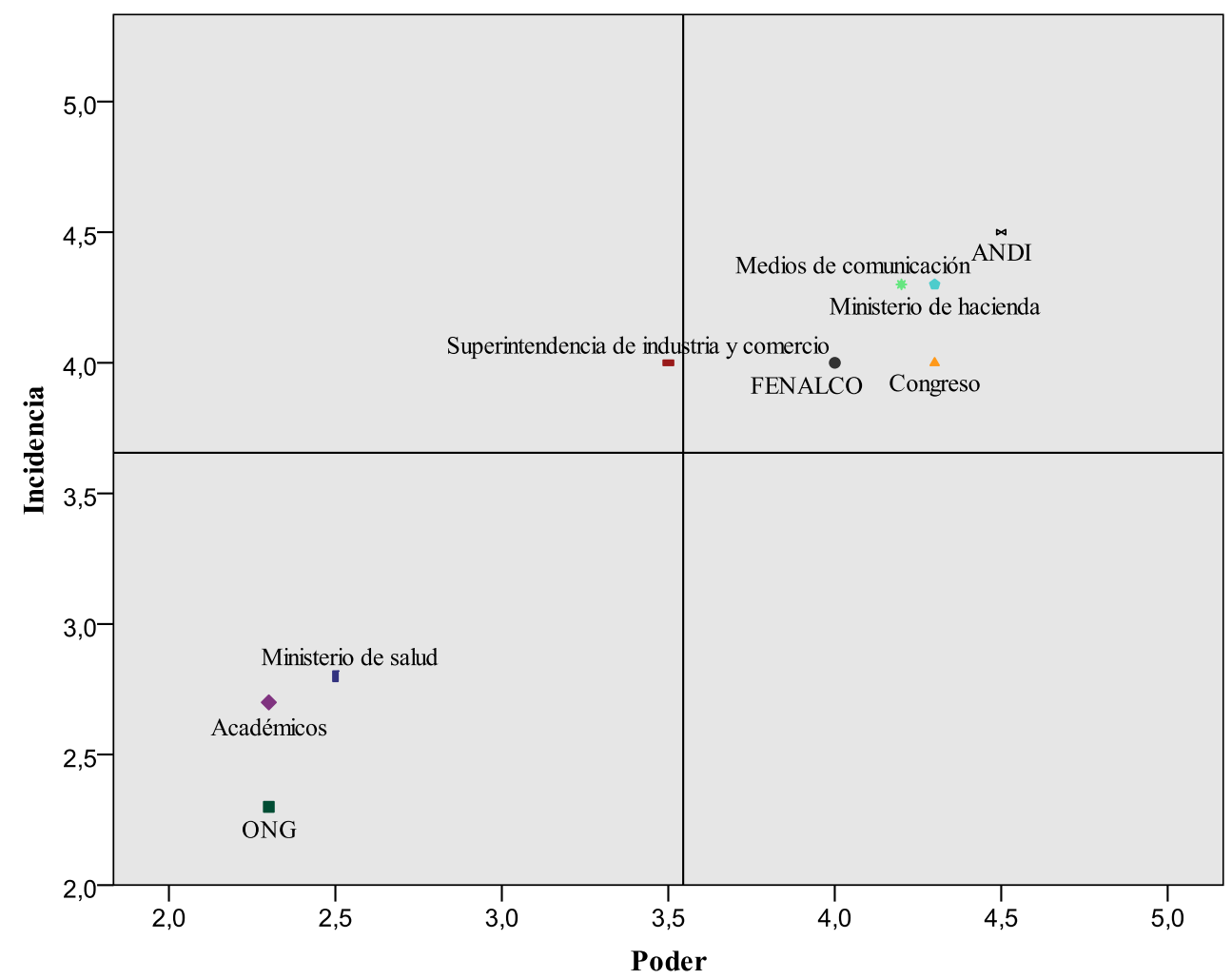

Grupos de interés

Fuente: elaboración propia. 
Figura 6. Mapa de actores, incidencia y poder en el escenario ideal de toma de decisiones

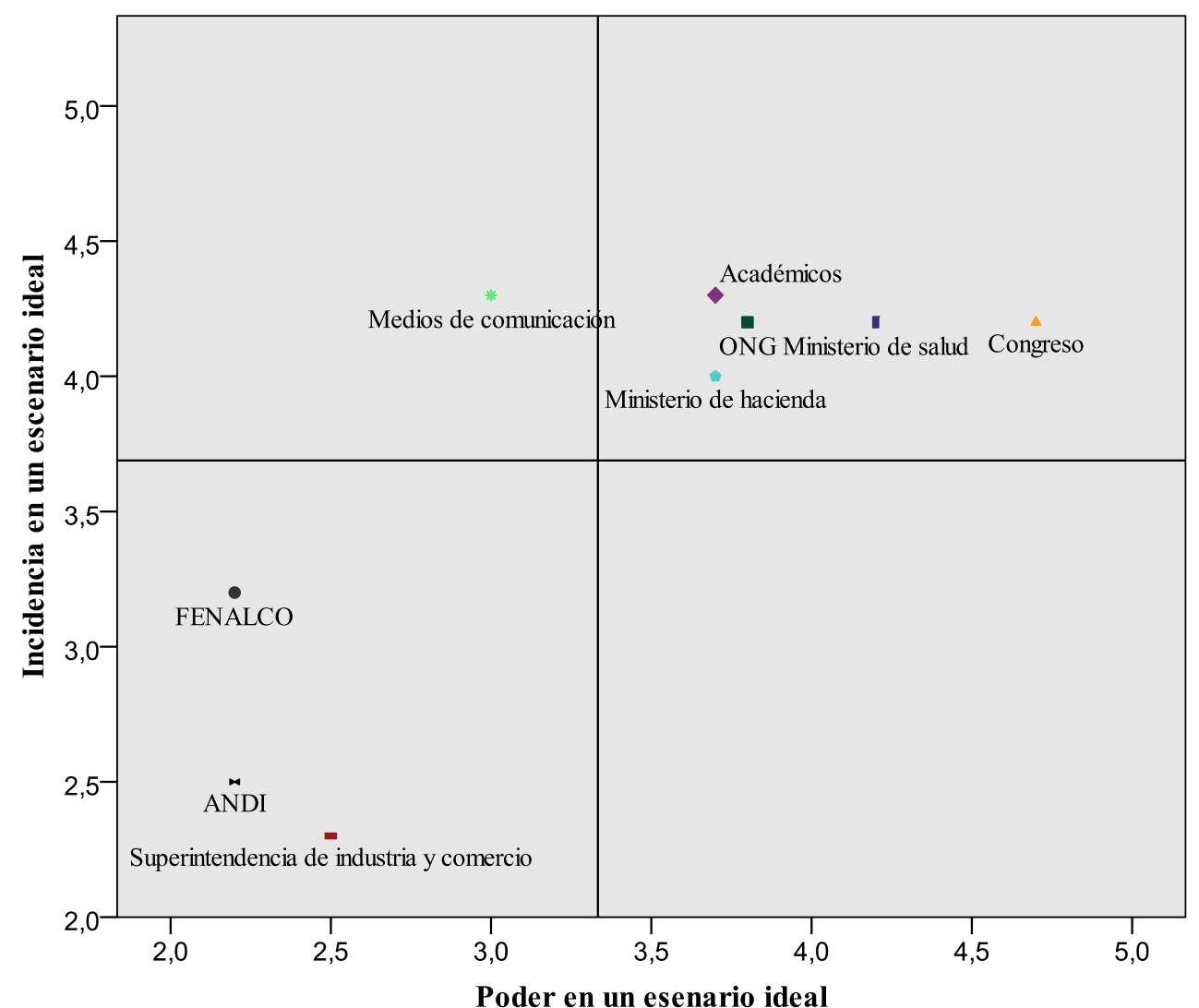

\section{Grupos de interés}

Fuente: elaboración propia.

\section{Discusión final}

Los hallazgos de este trabajo dan cuenta de la capacidad de la industria en el país para bloquear procesos de agenda pública que van en contra de sus intereses. Se evidenció incidencia y poder de grupos de interés como la ANDI y Fenalco, además de los actores institucionales, medios de comunicación, entre otros, que apoyaron y validaron su accionar. Así, Colombia se convirtió 
Díaz-García, Valencia-Agudelo, Carmona-Garcés y González-Zapata: Grupos de interés...

en el único país que propone esta medida tributaria para afrontar un problema grave de salud pública como el expuesto, pero no la somete a consideración del Poder Legislativo en pleno.

También es importante resaltar la brecha en la fase predecisional del ciclo de políticas públicas frente a la definición del problema, ya que los diferentes grupos de interés reconocen la magnitud de éste, pero las posibles soluciones aluden más a su rol o intereses que al bienestar general de la población. Es así como la industria, en su lógica de mercado, concibe un accionar de su parte basado en la oferta y la demanda, acompañado de estrategias de prevención que lleven a un consumo responsable de productos poco saludables como las bebidas azucaradas, mientras que el sector académico y las ONG hacen abogacía por un rol del Estado regulatorio y normativo que determine el tipo de producción y oferta que la industria hace a sus consumidores, y permita corregir las externalidades negativas, como las enfermedades asociadas a su consumo y los elevados costos que traen para el sistema de salud.

Estas dos visiones ponen de manifiesto las dificultades propias de una economía mixta, en la cual constantemente se tienen que definir las fronteras entre las actividades públicas y privadas, y la idea subyacente del rol que debe cumplir el Estado frente a la libre empresa y el control estatal de la producción, planteado por diferentes teóricos a lo largo de la historia (Stiglitz, 2000, pp. 12-14).

Con respecto al uso de los instrumentos de poder utilizados durante el proceso decisorio, se observó que la industria, acompañada del gremio de comerciantes en cabeza de Fenalco, utilizaron diferentes instrumentos para penetrar la agenda pública, tales como las capacidades organizativas y de persuasión, el poder económico, la influencia a través de la influjo en la reglamentación, entre otros. Los anteriores instrumentos se evidenciaron inequitativos, por ejemplo, al tener un mayor protagonismo en diferentes medios de comunicación para desestimar la pertinencia del impuesto, incidir para que la SIC censurara el comercial "Cuida tu vida, tómala en serio", disponer la presencia permanente de cincuenta y seis lobistas en el Congreso de la República desde que se realizó la propuesta al impuesto para presentarles a los parlamentarios los argumentos de la industria, entre otros. 
De acuerdo con Lindblüm (1991), la amplia gama de instrumentos de intervención y de reglas informales que utilizan tanto el sector público como los grupos de interés privado antes de tomar una decisión pública pueden provocar que esta no se dé exclusivamente mediante la autoridad competente; es decir, "la política de los grupos de interés se convierte en una especie de juego en el que los líderes de los grupos de interés y los decisores de políticas públicas actúan bajo ciertas reglas” (p. 113). Al respecto, Subirats (1994), a partir del carácter polisémico de la política, menciona que es necesario conocer "qué actores (políticos, burócratas, actores privados) han intervenido en la formulación y actuación de la política concreta planteada, qué distintas racionalidades de acción y de intereses utilizan, y contrastar los resultados con los objetivos finalmente alcanzados" (p. 41).

Frente a la incidencia y el poder de los diferentes grupos de interés, los hallazgos de este trabajo coinciden con la necesidad de que exista una reivindicación de la sociedad civil en el sistema democrático actual. Estos grupos, como se esbozó en el marco teórico, deben trabajar y fortalecer los mecanismos para que sus intereses queden inscritos en la agenda, o si estos ya están, para que permanezcan y se prioricen (Valencia, 2012). Sin embargo, tal y como lo mostró el escenario ideal de toma de decisiones, el poder de la decisión final, al igual que para grupos de interés privados, debe ser modesto (Smith, 1997).

Como perspectiva para futuros análisis, se reconoce la importancia de ampliar las entrevistas a más actores por sector para tener diferentes visiones y enriquecer el análisis, debido a que en un grupo de interés como el Congreso y demás grupos institucionales coexisten diferentes posiciones con respecto a aspectos como el abordado en este trabajo. Así mismo, se reconoce la importancia de ahondar en los métodos utilizados para la investigación de grupos de interés, ya que en la revisión de la literatura se observa que no hay un consenso en la metodología y en este caso en particular se conjugaron varias herramientas para potencializar el análisis (Abitbol \& Botero, 2005; Vargas, 2008).

En conclusión, la toma de decisiones se da en función de la movilización de los distintos intereses sectoriales y consiste en un enjeu de poder. Este 
Díaz-García, Valencia-Agudelo, Carmona-Garcés y González-Zapata: Grupos de interés...

caso refleja la necesidad del fortalecimiento del sistema democrático, con garantías a los procesos legislativos, y la creación de un ambiente político favorable, transparente, sin el uso de herramientas de influencia que puedan bloquear la agenda. Además, pone de manifiesto la importancia de una mayor incidencia de la sociedad civil, en cabeza de cualquier organización que la represente, en pro de favorecer no solo la fase de establecimiento de agenda y definición del problema público, sino todo el ciclo de las políticas públicas en las subsecuentes fases.

\section{Referencias}

Abitbol, P. \& Botero, F. (2005). Teoría de elección racional: estructura conceptual y evolución reciente. Colombia Internacional, 62, 132-145.

Aguilar, L. (2015). El concepto de política pública. En J. Cuervo (Ed.), Ensayos sobre politicas públicas (pp. 35-86). Bogotá: Universidad Externado de Colombia.

ANDI (Asociación Nacional de Empresarios de Colombia) (19 de mayo de 2016). Industria de bebidas no alcohólicas firma compromisos de autorregulación responsable. Boletín de prensa de Postobón. http://www.po stobon.com/sites/default/files/boletin_prensa_compromisos_vf_0.pdf

Bachrach, P. \& Baratz, M. (1963). Decisions and Non Decisions. American Political Science Review, 57(3), 632-642.

Banco Mundial (2003). Guía del usuario para el análisis del impacto social y en la pobreza. The International Bank for Reconstruction and Development. https://www.alcobendas.org/recursos/doc/Cooperacion/373266862_2 6102012133350.pdf

Bustamante, C. (2014). Los colombianos se toman $\$ 12,5$ billones en gaseosas y jugos. Portafolio. http://www.portafolio.co/negocios/empresas/colomb ianos-toman-12-billones-gaseosas-jugos-55860

Centro de Análisis Político Universidad EAFIT (2012). Mapa de actores para la construcción de una política pública de trabajo decente en 
Medellín. En J. Rosado-Duque y L. Tobón-Vallejo (Eds.), Medellín construye trabajos decentes (pp. 135-202). Medellín: Escuela Nacional Sindical.

Chávez, E. (2006). Ética en el Poder Legislativo. Boletín Mexicano de Derecho Comparado. 39(115), 93-124.

Congreso de la República de Colombia (2016). Proyecto de ley "por medio de la cual se adopta una reforma tributaria estructural, se fortalecen los mecanismos para la lucha contra la evasión y la elusión fiscal, y se dictan otras disposiciones". Impuesto nacional al consumo de bebidas azucaradas. http://www.minhacienda.gov.co/HomeMinhacienda/ShowProperty?n odeId=\%2FOCS\%2FP_MHCP_WCC-059074\%2F\%2FidcPrimaryF ile\&revision=latestreleased

Dusso, A. (2010). Legislation, Political Context, and Interest Group Behavior. Political Research Quarterly, 63(1), 55-67. https://doi.org/10.1177/1065 912908324202

Educar Consumidores (2017). Interferencia de la industria al impuesto a las bebidas azucaradas. Bogotá: Difundir Ltda.

Garcia, M., Villar-Uribe, M. \& Iunes, R. F. (2017). The political economy of the 2016 tobacco and proposed sugar-sweetened beverage tax increases in Colombia. Bogotá: World Bank Group.

Halpern, C. (2016). decisión. En L. Boussaguet, S. Jacquot y P. Ravinet, Diccionario de politicas públicas (pp. 194-203). Bogotá: Universidad Externado de Colombia.

Izquierdo, B. (2008). De la evaluación clásica a la evaluación pluralista. Criterios para clasificar los distintos tipos de evaluación. EMPIRIA, Revista de Metodología de las Ciencias Sociales, 16, 118-130.

Lindblüm, C. (1991). El proceso de elaboración de políticas públicas. México: Grupo Editorial Miguel Ángel Porrúa.

McKay, A. \& Webbs, S. (2007). Interest Group Competition on Federal Agency Rules. American Politics Research, 35(3), 336-357. https://doi. org/10.1177/1532673X06296571 
Díaz-García, Valencia-Agudelo, Carmona-Garcés y González-Zapata: Grupos de interés...

Medina, I. (2009). Cómo medir la influencia de los grupos de interés (propuestas desde el pluralismo, el elitismo y el nuevo institucionalismo) (Working Papers, 279), 1-70. Institut de Ciéncies Polítiques i SocialsICPS. https: //www.icps.cat/archivos/WorkingPapers/wp279.pdf?noga $=1$

Ministerio de Hacienda y Crédito Público (2016). Exposición de motivos del proyecto de ley "por medio de la cual se adopta una reforma tributaria estructural, se fortalecen los mecanismos para la lucha contra la evasión y la elusión fiscal, y se dictan otras disposiciones". Bogotá: Ministerio de Hacienda y Crédito Público. http://www.minhacienda.gov.co/HomeM inhacienda/ShowProperty? nodeId=\%2FOCS\%2FP_MHCP_WCC-0 59072\%2F\%2FidcPrimaryFile\&revision=latestreleased

MPS (Ministerio de la Protección Social), INS (Instituto Nacional de Salud) \& ICBF (Instituto Colombiano de Bienestar Familiar) (2010). Encuesta Nacional de la situación nutricional en Colombia 2010 ENSIN. Bogotá: Da Vince Editores \& CIA.

Moodie, R., Stuckler, D., Monteiro, C., Sheron, N., Neal, B., Thamarangsi, T., Lincoln, P. \& Casswell, S. (2013). Profits and pandemics: prevention of harmful effects of tobacco, alcohol, and ultra-processed food and drink industries. The Lancet, 381(9867), 670-679.

Nestle, M. (2015). Soda politics: Taking on big soda (and winning). EE.UU.: Oxford University Press.

OMS (Organización Mundial de la Salud) (2015). Directriz: Ingesta de azúcares para adultos y niños. http://apps.who.int/iris/bitstream/10 665/154587/2/WHO_NMH_NHD_15.2_spa.pdf?ua=1

OPS (Organización Panamericana de la Salud) (2015). Experiencia de México en el establecimiento de impuestos a las bebidas azucaradas como estrategia de salud pública. México D.F.: OPS. http://iris.paho.org/xmlui/handle /123456789/18390

Pagliari, S. \& Young, K. (2016). The interest ecology of financial regulation: interest group plurality in the design of financial regulatory policies. Socio-Economic Review, 2(14), 309-337. 
Popkin, B. \& Hawkes, C. (2015). Sweetening of the global diet, particularly beverages: patterns, trends, and policy responses. The Lancet Diabetes \& Endocrinology, 4(2), 174-186. http://dx.doi.org/10.1016/S2213-858 7(15)00419-2

Saurugger, S. (2016). Grupos de interés. En L. Boussaguet, S. Jacquot y P. Ravinet, Diccionario de politicas públicas (pp. 326-333). Bogotá: Universidad Externado de Colombia.

Singh, G., Micha, R., Khatibzadeh, S., Lim, S., Ezzati, M. \& Mozaffarian, D. (2015). Estimated Global, Regional, and National Disease Burdens Related to Sugar-Sweetened Beverage Consumption in 2010. Circulation, 132(8), 639-666. https://doi.org/10.1161/CIRCULATIONAHA.114 .010636

Smith, M. (1997). El pluralismo. En D. Marsh, y G. Stoker (Eds.), Teoría y métodos de la ciencia politica (pp. 217-234). Madrid: Alianza Editorial.

Stiglitz, J. (2000). El sector público en una economía mixta. En La economía del sector público (pp. 11-31). Barcelona: Antoni Bosch.

Subirats, J. (1994). Análisis de políticas públicas y eficacia de la administración. Madrid: Ministerio para las administraciones públicas.

Tapia, M., Campillo, B., Cruickshank, S. \& Morales, G. (2010). Manual de incidencia en politicas públicas. http://www.alternativasycapacidades.org /sites/default/files/MIPP.pdf

Truman, D. (1951). The governmental process. New York: Knopf.

Valencia, G. (2005). Los grupos de interés en la regulación de la industria eléctrica colombiana. Lecturas de Economía, 62, 123-156.

Valencia, G. (2012). Incidencia de la sociedad civil en el ciclo de las políticas públicas. Papel Politico, 17(2), 469-496.

Vargas, J. (2008). La alternancia desde el pluralismo democrático. Revista de Derecho, 20, 1-16. 
Díaz-García, Valencia-Agudelo, Carmona-Garcés y González-Zapata: Grupos de interés...

Victor, J. (2007). Strategic Lobbying Demonstrating How Legislative Context Affects Interest Groups' Lobbying Tactics. American Politics Research, 35(6), 826-845. https://doi.org/10.1177/1532673X07300681

Vidal, G. (2008). La teoría de la elección racional en las ciencias sociales. Revista Sociológica, 67(23), 1-16. 